\title{
Characteristics of gut microbiota and its response to a Chinese Herbal Formula in elder patients with metabolic syndrome
}

\author{
Yongcheng $\mathrm{Ni}^{1}$, Chunlong $\mathrm{Mu}^{2}$, Xiangyu $\mathrm{He}^{2}$, Kaiming Zheng ${ }^{1}$, Hongmin Guo ${ }^{1, *}$, Weiyun $\mathrm{Zhu}^{2, *}$ \\ ${ }^{1}$ Geriatrics Department, Affiliated Hospital of Nanjing University of Traditional Chinese Medicine, Nanjing, China; \\ ${ }^{2}$ Jiangsu Key Laboratory of Gastrointestinal Nutrition and Animal Health, Laboratory of Gastrointestinal Microbiology, College \\ of Animal Science and Technology, Nanjing Agricultural University, Nanjing, China.
}

\begin{abstract}
Summary Alterations in gut microbiota have been known to play a critical role in metabolic syndrome. However, the microbial features in elderly patients with metabolic syndrome remain unclear. A traditional Chinese Herbal Formula, Yangyin Tiluo Decoction (YTD), can alleviate metabolic syndrome and cardiovascular disease. To characterize gut microbiota in elder patients and effects of YTD on gut microbiota during treatment of metabolic syndrome, 11 healthy elderly persons and 12 elderly persons (aged 60-90 years) with metabolic syndrome were enrolled. The patients were randomly assigned to receive YTD for 4 weeks $(200 \mathrm{~mL}$ of the decoction two times daily). The microbial composition in healthy control, pre- and postYTD treatment group were analyzed by $16 \mathrm{~S}$ rRNA sequencing of fecal DNAs. Biochemical measurements were conducted for elderly patients. The results showed a high interindividual variation of gut microbiota in elderly persons. The gut microbiota was dominated by phylum Firmicutes and Actinobacteria, which was distinct from the previously defined microbiota in Irish elderly persons. The elderly patients with metabolic syndrome had higher proportions of Lactobacillus and Bifidobacterium, and lower proportions of Anaerostipes, Coprococcus, Ruminococcus than healthy controls. YTD treatment reduced the abundance of genus Bacteroidales Incertae Sedis and species Enterobacteriaceae Incertae Sedis. The concentration of plasma lipoprotein (a) was also reduced, which was negatively correlated with the abundance of an Acinetobacter species. These results reveal a remarkable dominance of Firmicutes and Actinobacteria, and highlight the distinct gut microbiota in elderly patients with metabolic syndrome, which may be involved in pathogenesis. Furthermore, the benefits of YTD treatment were observed, providing an approach to improve metabolic syndrome in elderly patients.
\end{abstract}

Keywords: Lactobacillus, metabolic syndrome, elderly persons, Chinese Herbal Formula treatment

\section{Introduction}

Metabolic syndrome (visceral obesity, dyslipidemia,

\footnotetext{
*Address correspondence to:

Dr. Hongmin Guo, Geriatrics Department, Affiliated Hospital of Nanjing University of Traditional Chinese Medicine, No. 155, Hanzhong Road, Qinhuai District, Nanjing, Jiangsu, China.

E-mail: babyfishme@163.com

Dr. Weiyun Zhu, Jiangsu Key Laboratory of Gastrointestinal Nutrition and Animal Health, Laboratory of Gastrointestinal Microbiology, College of Animal Science and Technology, Nanjing Agricultural University, No. 6, Tongwei Road, Nanjing, Jiangsu, China.

E-mail: zhuweiyun@njau.edu.cn
}

hyperglycemia, and hypertension), has become one of the major public-health challenges worldwide (1), especially in elderly persons. The pathogenesis, unified by the putative mechanism of insulin resistance, was thought to be related to interactions between sedentary lifestyle, diet, and genetic factors (2). Due to the decline in body function, elderly persons are vulnerable to cardiovascular disorders. Therefore, the etiology of metabolic syndrome can be more complex in elderly persons.

Gut microbiota may have a vital role in metabolic syndrome. The alteration in composition and activity of gut microbiota affects the pathogenesis of obesity and related disorders (3). Accumulating evidence suggests that dysbiosis of gut microbiota induced by a high fat/ high calorie diet has a key role in the development 
of obesity, insulin resistance and other hallmarks of metabolic syndrome (4). Furthermore, reductions in Bifidobacterium, butyrate-producing bacteria and increases in pro-inflammatory/pathogenic bacteria are always associated with the development of metabolic syndrome in humans (5). Defining the gut microbiota in elder persons with metabolic syndrome can provide reference for possible therapies that target gut microbiota. However, the composition of gut microbiota in elder patients with metabolic syndrome remains unclear.

To improve the quality of life in elderly persons with metabolic syndrome, drugs that treat cardiovascular diseases may be useful. Among the treatments, Chinese Herbal Formulas (TCMs) can serve as valuable therapeutic strategies and drug discovery resources (6). TCMs, which are forms of polypharmacy, have been clinically used in China for treatment of many diseases for thousands of years (7).

Yangyin Tiluo Decoction (YTD) is used to treat cerebral infarction in elders in China. Clinical studies found that YTD could alleviate metabolic syndrome in elderly persons (8). YTD treatment could also downregulate the expression of tumor necrosis factor $\alpha$ and interleukin 6 in lipopolysaccharide-treated human venous endothelial cells (9), supporting its function in alleviating cardiovascular diseases. It also suggested that the excess caloric consumption driving the current epidemic of metabolic syndrome may be caused by alterations in host-microbiota interactions (10). Even though, whether YTD could modulate gut microbiota during treatment of metabolic syndrome is still unknown.

In this study, we conducted a controlled, preand post-treatment comparison trial to define the characteristics of gut microbiota related to metabolic syndrome in elderly persons. Furthermore, the structural alterations of gut microbiota in response to YTD were also investigated. These results provide reference for understanding gut microbiota in elderly persons with metabolic syndrome.

\section{Materials and Methods}

\subsection{Ethics, consent and permissions}

The study was approved by the Ethics Committee of Affiliated Hospital of Nanjing University of Traditional Chinese Medicine (No. 2013NL-029-02, registered 19 April 2013), and written informed consent was obtained from each participant before their admission to the protocol.

\subsection{Study design}

The study was a 4-week, pre- and post-treatment comparison trial with healthy controls. Participants were recruited by Geriatrics Department of Jiangsu Province
Hospital of Traditional Chinese Medicine from July 2013 to June 2015, during which the samples were collected. The authors had access to information that could identify individual participants during or after data collection.

Patient enrollment was conducted according to inclusion and exclusion criteria. The inclusion criteria were: $i$ ) previously diagnosed metabolic syndrome patients; ii) age of 60-89 years; iii) good compliance; iv) signed informed consent. Volunteers who met all four criteria were included as disease group, and those who met $i i)-i v$ ) were included as the healthy control group. Exclusion criteria were: $i$ ) the etiology was too complex to obtain clear evaluation about the efficiency and safety of the drug; ii) disability and psychiatric disturbance; iii) presence of cancer; $i v$ ) history of abdominal operation or intestinal organic lesion; v) allergic persons; $v i$ ) treatment with antibiotics, probiotics, antidiarrheal agent, or swelling agent within the past 1 month; vii) unitary or inappropriate diet structure, dietary intake much higher or lower than normal elder persons.

Metabolic syndrome was defined using the 2004 Chinese Diabetes Society criteria, according to the presence of three or more of following aspects: $i$ ) overweight and/or obesity: $\mathrm{BMI} \geq 25.0 \mathrm{~kg} / \mathrm{m}^{2} ; i i$ ) hyperglycemia: fasting plasma glucose $\geq 6.1 \mathrm{mmol} / \mathrm{L}$ and/or $2 \mathrm{~h}$ plasma glucose $\geq 7.8 \mathrm{mmol} / \mathrm{L}$, or previously diagnosed type 2 diabetes and receiving treatment; iii) systolic blood pressure/diastolic blood pressure $\geq$ $140 / 90 \mathrm{mmHg}$, or previously diagnosed hypertension and receiving treatment; $i v$ ) dyslipidemia: fasting plasma triglyceride $\geq 1.7 \mathrm{mmol} / \mathrm{L}(150 \mathrm{mg} / \mathrm{dL})$, and $/$ or fasting plasma HDL-c $<0.9 \mathrm{mmol} / \mathrm{L}$ (men) or $<1.0 \mathrm{mmol} / \mathrm{L}$ (women) (10).

Using the screening criteria, 11 healthy elderly people were assigned as the healthy control group, while 12 patients were treated with YTD for four weeks. The information of selected subjects can be found in Table 1. No missing data were observed for each variable of interest. All the participants were advised to take lowsalt, low-fat diabetic diet, and to avoid taking medication that may affect the gut microbiota, such as prebiotics and probiotics. The variables were included in data analysis to avoid biases. The study size was chosen based on the numbers of patients passing the inclusion criteria and the requirement for statistical analysis.

\subsection{Drug administration}

The Yangyin Tiluo Decoction (YTD) is a TCM composed of Polygonatum sibiricum (20 g), Lycium barbarum (15 g), Rehmannia glutinosa Libosch (12 g), Rhodiola rosea (20 g), Panax notoginseng (5 g), Ligusticum chuanxiong Hort (10 g), Lumbricus (10 g), Radix puerariae (20 $\mathrm{g})$, and Folium nelumbinis $(20 \mathrm{~g})$. The preparation of YTD was conducted based on the Standard Operating Procedure of Jiangsu Province Hospital of Traditional Chinese Medicine. Briefly, all the components were put 
Table 1. Characteristics of the study subjects

\begin{tabular}{lcc}
\hline Items & Healthy control & Patients with metabolic syndrome \\
\hline Age & $71.8 \pm 11.5$ & $82.9 \pm 3.3$ \\
$n$ & 11 & 12 \\
Gender (male/female) & $6 / 5$ & $8 / 4$ \\
Height $(\mathrm{cm})$ & $1.66 \pm 0.05$ & $7.68 \pm 0.04$ \\
Weight $(\mathrm{kg})$ & $65.9 \pm 5.0$ & $72.2 \pm 8.3$ \\
BMI $\left(\mathrm{kg} / \mathrm{m}^{2}\right)$ & $24.1 \pm 1.5$ & $25.6 \pm 2.7$ \\
$\quad>25$ & 4 & 7 \\
$\quad<25$ & 7 & 5 \\
\hline
\end{tabular}

Values of age, height, weight, and BMI are expressed as mean \pm SD.

into a ceramic pharmacy pot, and $300 \mathrm{~mL}$ cold drinking water was added to soak for 1 hour. After boiling with a raging blaze for $10 \mathrm{~min}$, it was kept boiling for $20 \mathrm{~min}$ with small fire. The decoction was filtered into $200 \mathrm{~mL}$ bags (part 1) while the decoction was hot. Then another $300 \mathrm{~mL}$ cold drinking water was added to the residue, and the boiling process was repeated. The decoction was filtered into another set of $200 \mathrm{~mL}$ bags (part 2). Part 1 and 2 were mixed homogeneously, then divided into two equal parts, and each part was taken after meals in the morning and evening respectively for 4 weeks.

\subsection{Sample collections}

For healthy groups, the first feces portion at morning was collected at the beginning of the experiment. For disease group, the first feces portion and fasting venous blood were sampled at the beginning of the experiment (before YTD treatment) and after YTD treatment for 4 weeks. Samples were kept at $-80^{\circ} \mathrm{C}$ before further analysis.

\subsection{Fecal DNA extraction and pyrosequencing}

All fecal samples from healthy and diseased persons were used for fecal DNA extraction, including 11 samples of healthy control, 12 samples of patients before YTD treatment, and 12 samples after YTD treatment. Total genomic DNA in feces were extracted from $0.3 \mathrm{~g}$ of sample using bead-beating and phenol-chloroform extraction according to Zoetendal et al. (11). The quality and concentration of DNA were evaluated using a NanoDrop ND-1000 spectrophotometer (NanoDrop ${ }^{\circledR}$ Technologies, Wilmington, DE, USA).

Amplification of genomic DNA was performed using barcoded primers that targeted the $\mathrm{V} 3$ to $\mathrm{V} 4$ regions of the bacterial 16S rRNA gene, with universal bacterial primers (forward 5'-barcode-TAC GGR AGG CAG CAG-3' and reverse 5'-AGG GTA TCT AAT CCT3 '), where barcode is an eight-base sequence unique to each sample. Procedures of amplification, amplicon purification, sequencing, and basic analysis were conducted according to previous study (12). Purified amplicons were pooled in equimolar and paired-end sequenced $(2 \times 250)$ on an Illumina MiSeq platform according to standard protocols. The raw reads were deposited into the NCBI Sequence Read Archive (SRA) database (accession SRP118482).

Raw fastq files were demultiplexed, quality-filtered using QIIME version 1.17 (13) with the following criteria: $i$ ) The 250 bp reads were truncated at any site receiving an average quality score $<20$ over a $10 \mathrm{bp}$ sliding window, discarding the truncated reads that were shorter than $50 \mathrm{bp}$; ii) exact barcode matching, 2 nucleotide mismatch in primer matching, reads containing ambiguous characters were removed; iii) Only sequences that overlaped longer than $10 \mathrm{bp}$ were assembled according to their overlap sequence. Reads which could not be assembled were discarded. Operational taxonomic units (OTUs) were clustered with a 97\% similarity cutoff using UPARSE (version 7.1 http://drive5.com/uparse/) and chimeric sequences were identified and removed using UCHIME. The phylogenetic affiliation of each 16S rRNA gene sequence was analyzed by Ribosomal Database Project Classifier (http://rdp.cme.msu.edu/) against the Silva (SSU123) 16S rRNA database using a confidence threshold of $70 \%$ (14). The rarefaction analysis, alpha diversity, and taxonomic assignment were also analyzed in QIIME. Unweighted Unifrac principal coordinate analysis (PCoA) (15) based on OTUs was performed to provide an overall view of the microbial structures.

\subsection{Biochemical measurements}

During the experiment, nine patients after YTD treatment finished the essential procedures for sampling fasting venous blood. Biochemical measurements of plasma glucose, total cholesterol, triglyceride, lipoprotein (a), low-density lipoprotein cholesterol, high-density lipoprotein cholesterol, and uric acid were conducted in the central laboratory of Jiangsu Province Hospital of Traditional Chinese Medicine (Nanjing, China). The biochemical parameters were analyzed using an automatic analyzer (Beckman AU5800; Beckman Coulter, USA). Lipoprotein (a) is a useful indicator in patients with cerebrovascular disease and atherosclerosis.

\subsection{Statistical analysis}

Homogeneity of variance was tested for all measures. 
To characterize the gut microbiota in elder persons with metabolic syndrome, microbiome data from patients before YTD treatment were analyzed against healthy persons and submitted for LEfSe analysis. LEfSe algorithm can identify differentially abundant features between two or more biological conditions (16). This method defines discriminative features based on the following conditions: $i$ ) the alpha value for the factorial Kruskal-Wallis test among classes is $<0.05$ and $i$ ) the threshold on the logarithmic linear discriminant analysis (LDA) effect sizes for is $>2.0$. LEfSe was performed on the website http:// http://huttenhower.org/galaxy.

To compare the effect of YTD treatment, biochemical measurement data and microbiome were analyzed using paired Wilcoxon test with modules implemented in MetaboAnalyst version 3.0 (17). Significance was considered at $p<0.05$. In case of multiple comparisons, $p$-values were adjusted with a false discovery rate (FDR) analysis (18), limiting the overall false discovery rate to $5 \%(q<0.05)$. Adjustments of comparisons for age and gender were performed using analysis of covariance (SPSS verson 20.0; SPSS Inc., Chicago, Illinois).

Correlations between microbial composition with biochemical parameters were further analyzed using Spearman's rho correlation analysis (GraphPad Prism version 6.0, GraphPad Software, San Diego, CA). Correlation was considered significant when the absolute value of Spearman's rank correlation coefficient (Spearman's rho) was above 0.6 and statistically significant $(p<0.05)$.

\section{Results}

\subsection{Summary of pyrosequencing data}

In total $1,281,404$ qualified bacterial $16 \mathrm{~S}$ rRNA gene reads were obtained from 35 fecal samples $(36,611$ reads per sample) after pyrosequencing and used for subsequent analysis. The rarefaction analysis including all samples revealed a curve approaching saturation (Figure S1, http://www.ddtjournal.com/action/ getSupplementalData.php?ID=25), indicating that the measurement at the threshold level covers almost the full extent of the microbial diversity. The abundancebased coverage was higher than 0.99 for all three groups (Table S1, http://www.ddtjournal.com/action/ getSupplementalData.php?ID=25). The estimators of microbial diversity and richness, including Chaol index, and Shannon and Simpson diversity index, was not significantly different between groups ( $p>$ 0.05, Table S1, http://www.ddtjournal.com/action/ getSupplementalData.php?ID=25).

\subsection{High inter-individual variation of the elder gut microbiota}

OTU-based unweighted principal coordinates analysis showed that the overall structures were not significantly different between groups $(p>0.05)$. We noted the scattered distribution of different samples in healthy elderly persons (Figure 1A). Taxonomy-based analysis showed that the relative abundance of Bacteroidetes ranged from $0.27 \%$ to $50.9 \%$ and that the relative abundance of Actinobacteria ranged from $0.44 \%$ to $31.4 \%$, which indicated a high inter-individual variation of gut microbiota in elderly persons (Figure 1B).

The unweighted PCoA showed that the response to YTD treatment varied between patients. Sample IDs 2, 6, 7, and 10 showed a huge shift in microbial composition while others showed a slight shift (Figure 1A), suggesting an individual-specific response to YTD treatment.

\subsection{Firmicutes- and Actinobacteria-dominant gut microbiota in the elder cohort}

At the phylum level, a total of 14 phyla were observed. In the cohort, Firmicutes was the most dominant phylum with an average abundance range from 52.3\% to $72.8 \%$ (Figure 2A). In patients before and after YTD treatment, Actinobacteria was also dominant, which contributed $22.2 \%$ and $22.4 \%$ of gut microbiota before or after YTD treatment respectively. Bacteroidetes
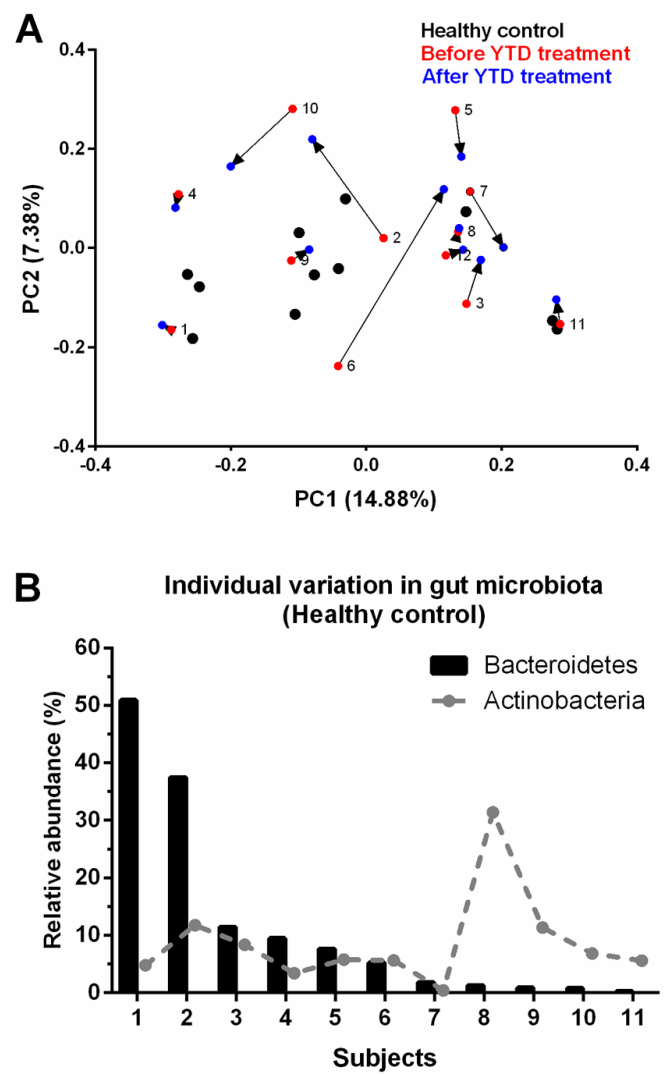

Figure 1. Principle coordinate analysis by unweighted Unifrac distance (A) and individual variation in the abundances of Bacteroidetes and Actinobacteria in healthy controls (B). Abbreviations used: YTD, Yangyin Tiluo Decoction. 
A

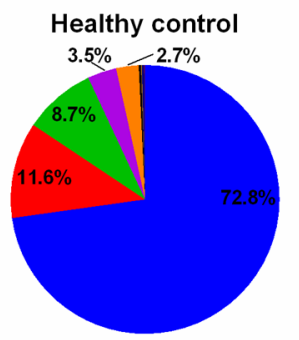

Before YTD treatment

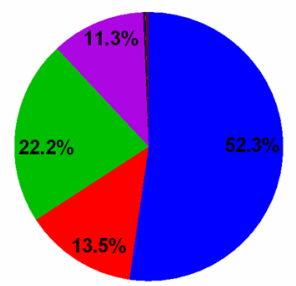

B

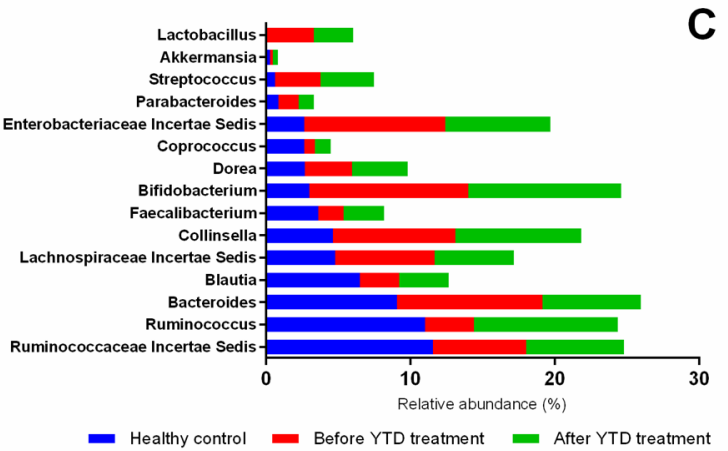

After YTD treatment

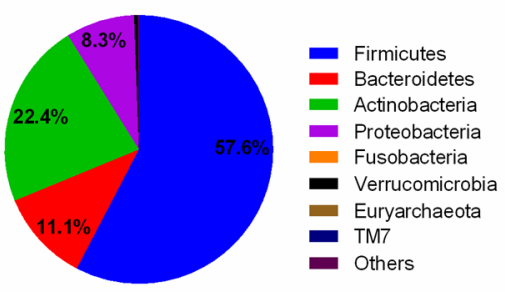

C

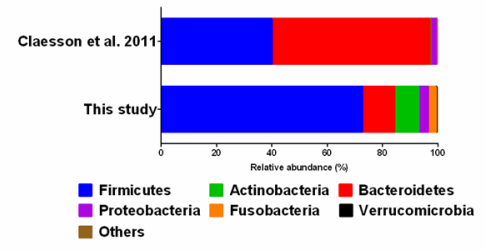

Figure 2. Fecal microbiota composition at phylum (A) and genus (B) level from elderly persons. (C) Comparison of microbiota composition with studies of Irish elderly population. Abbreviations used: YTD, Yangyin Tiluo Decoction.

constituted the next dominant phylum with the average abundance range from $11.1 \%$ to $13.5 \%$. The Firmicutesand Actinobacteria- dominant microbiota were also apparent at the genus level, as reflected by the dominant genus Ruminococcaceae Incertae Sedis, Ruminococcus, Bifidobacterium, and Collinsella (Figure 2B). When comparing the microbial composition between studies (Figure 2C), the dominant phylum was Firmicutes (average abundance 57\%) followed by Bacteroidetes (average abundance 42\%) in an Irish cohort (19), which was quite different from the healthy control group and patient group in the present study.

\subsection{Characteristics of fecal microbiota in elder patients with metabolic syndrome}

By using LEfSe analysis, a total of 24 microbial features were found to be different between healthy controls and elderly patients with metabolic syndrome before YTD treatment, with nine enriched in metabolic syndrome patients and 15 enriched in healthy controls (Figure 3). The fecal microbiota of untreated metabolic syndrome patients exhibited a relative abundance of Actinobacteria (Bifidobacteriaceae, Bifidobacterium, Bifidobacteriaceae Incertae Sedis), Lactobacillaceae (Lactobacillus), and Megasphaera compared to healthy controls. Interestingly, the relative abundance of Lactobacillus was increased by 187 -fold in untreated metabolic syndrome patients $(3.28 \%)$ compared to untreated metabolic syndrome patients $(0.018 \%)$. On the contrary, metabolic syndrome patients had a lower abundance of phylum Firmicutes, families Erysipelotrichaceae, Clostridiaceae, and Turicibacteraceae, genus

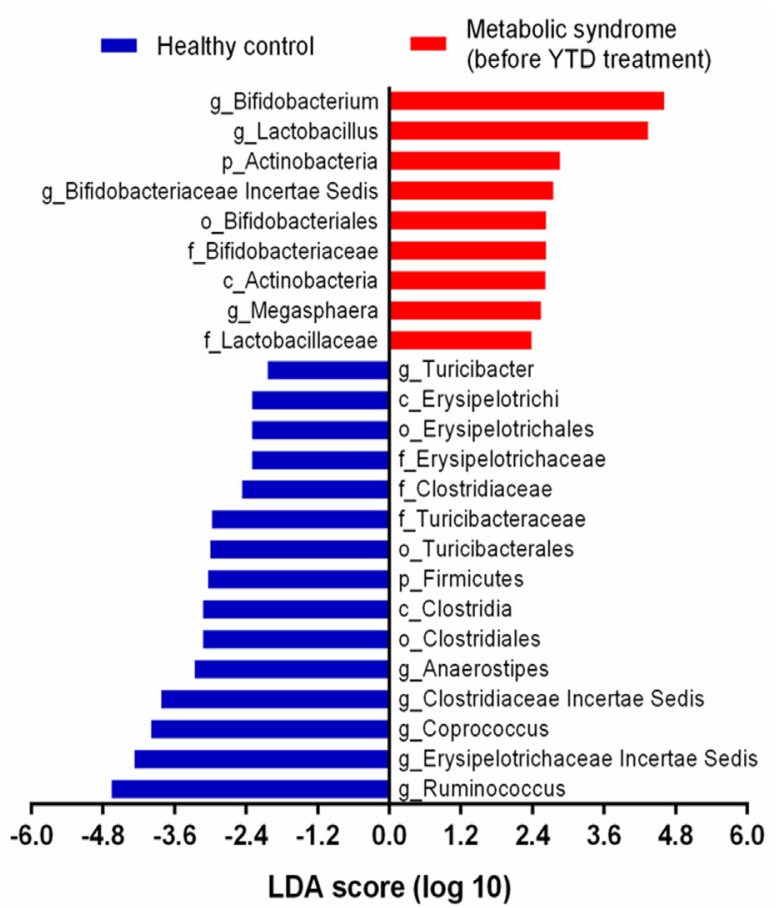

Figure 3. Differences in the gut microbiota composition between healthy controls and elderly patients with metabolic syndrome (before YTD treatment). Abbreviations used: YTD, Yangyin Tiluo Decoction.

Turicibacter, Anaerostipes, Ruminococcus, Coprococcus, Clostridiaceae Incertae Sedis, and Erysipelotrichaceae Incertae Sedis than healthy controls. The detailed results of relative abundances and LDA scores can be found in Table S2 (http://www.ddtjournal.com/action/ getSupplementalData.php?ID=25). 


\subsection{Effects of YTD treatment on gut microbiota and biochemical parameters}

Compared with the untreated patients, YTD treatment increased the relative abundance of Moraxellaceae, Acinetobacter, species Acinetobacter Incertae Sedis and Erysipelotrichaceae Incertae Sedis, while it decreased the relative abunance of Alphaproteobacteria, Rhizobiales, genus Bacteroidales Incertae Sedis, and species Enterobacteriaceae Incertae Sedis $(p<0.05$, Figure 4A). The abundance of species Acinetobacter Incertae Sedis and Erysipelotrichaceae Incertae Sedis was increased by 18.9 -fold and 86.7 fold, respectively. The abundance of genus Bacteroidales Incertae Sedis and species Enterobacteriaceae Incertae Sedis were decreased by 21.5-fold and 2.47-fold, respectively.

Analysis of plasma parameters indicated that YTD treatment reduced the concentrations of lipoprotein (a) $(p<0.05$, Figure 4B, Table 2), while it did not affect the concentrations of glucose, cholesterol, triglyceride, low-density lipoprotein cholesterol, highdensity lipoprotein cholesterol, and uric acid $(p>0.05$, Table 2). Correlation analysis identified the significant negative correlation between the concentrations of lipoprotein (a) with species Acinetobacter Incertae Sedis (Figure 4C).

\section{Discussion}

Changes in gut microbiota have been associated with the presence of metabolic syndrome. In the present study, by analyzing microbiota composition in a Chinese elderly population, we found that Chinese Herbal Formula YTD altered microbial composition and decreased metabolic markers of cerebrovascular disease in metabolic syndrome patients. These results are of great significance for targeting gut microbiota to improve health in elderly persons.

\subsection{Characteristics of gut microbiota in the Chinese elderly populations: inter-individual variability}

Employing the Chinese elderly population, we clearly showed high inter-individual variability of gut microbiota between subjects. These features have also been found in healthy Irish elderly populations (19) or hospitalized Italian elderly populations (20). Multiple factors, such as diet, physiological condition, and age, can increase the inter-individual variability of gut microbiota. Accompanying the individualized microbiota observed, the response of gut microbiota to YTD treatment was also individual-specific. It cannot be determined which factor contributes to the outcome. However, this
A

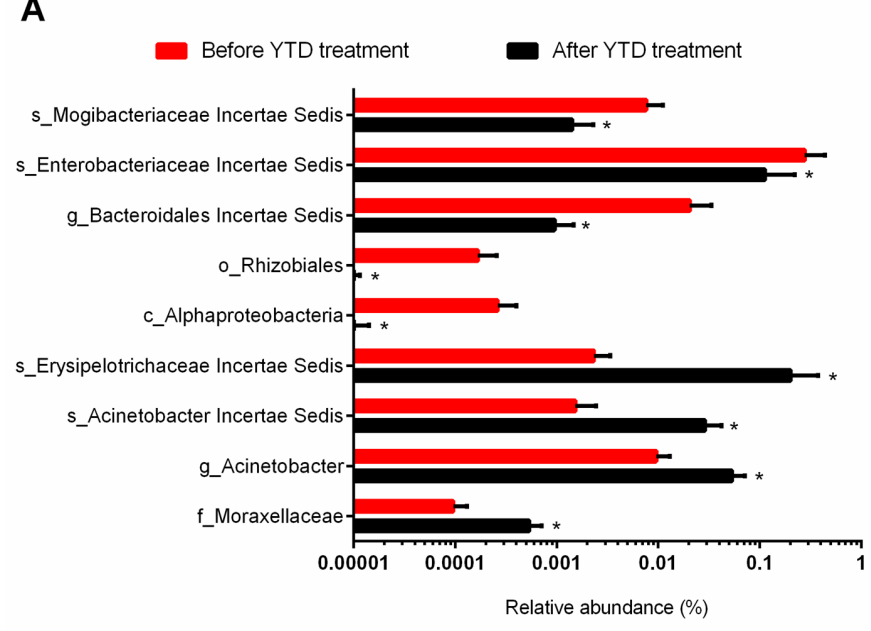

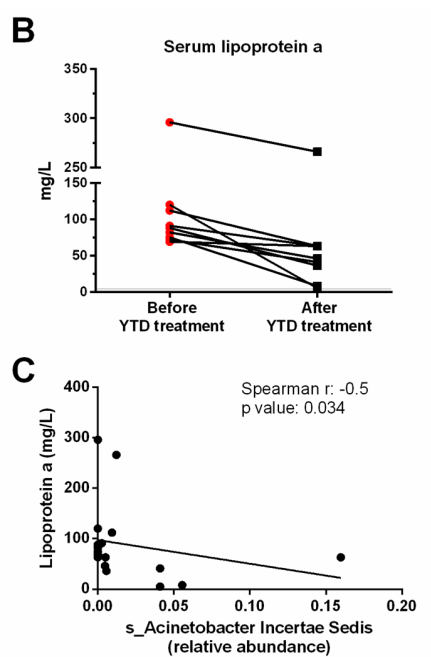

Figure 4. Effects of YTD treatment on the microbial composition (A) and plasma lipoprotein (B). (C) Correlations between significantly changed taxa with lipoprotein (a). Abbreviations used: YTD, Yangyin Tiluo Decoction.

Table 2. Plasma biochemical parameters in elder patients

\begin{tabular}{lcc}
\hline Items & Before YTD treatment & After YTD treatment \\
\hline Glucose $(\mathrm{mmol} / \mathrm{L})$ & $5.96(4.54-8.03)$ & $6.98(3.66-10.18)$ \\
Cholesterol $(\mathrm{mmol} / \mathrm{L})$ & $3.41(2.22-4.96)$ & $3.22(2.38-5)$ \\
Triglyceride $(\mathrm{mmol} / \mathrm{L})$ & $1.05(0.65-2.13)$ & $0.98(0.65-2.16)$ \\
Lipoprotein $(\mathrm{a})(\mathrm{mg} / \mathrm{L})$ & $88(69-296)$ & $46(5-266)$ \\
Low-density lipoprotein cholesterol $(\mathrm{mmol} / \mathrm{L})$ & $1.95(1.08-2.97)$ & 0.747 \\
High-density lipoprotein cholesterol $(\mathrm{mmol} / \mathrm{L})$ & $1.03(0.74-1.36)$ & 0.741 \\
Uric acid $(\mu \mathrm{mol} / \mathrm{L})$ & $348.3(214.2-462.9)$ & 0.002 \\
\hline
\end{tabular}

Abbreviations used: YTD, Yangyin Tiluo Decoction. 
phenomenon highlights the importance of considering individualized treatment for modulating gut health.

\subsection{Increase of lactic acid bacteria and decrease of butyrate-producing bacteria in elderly patients with metabolic syndrome}

A novel finding of the present study was that the elderly patients with metabolic syndrome had higher proportions of Lactobacillus and Bifidobacterium, and lower proportions of butyrate-producing bacteria than healthy controls. Lactobacillus and Bifidobacterium are generally considered to be health-promoting, and some species are widely used as probiotics (21). The abundances of Lactobacillus were also increased in type 2 diabetic patients $(60 \pm 8$ years old) compared with healthy controls $(40 \pm 8$ years old $)$ in a South China population (22). An increase in Lactobacillus and a decrease in butyrate-producing bacteria have been also observed in type 2 diabetes patients $(23,24)$, inflammatory bowel disease patients (25), and highfat diet-induced fatty liver mice (26). Interestingly, both diabetes and inflammatory bowel disease patients had an elevated pro-inflammation response in vivo. In elderly persons, the predisposition to pro-inflammation response can be easier in gut due to the aging process (27). Lactobacillus species, such as Lactobacillus plantarum could induce interleukin $1 \beta$ production and increase the inflammatory response (28). A previous study suggested that in metabolic syndrome patients, an increase in intestinal glucose availability may raise the substrate for Lactobacillus (23). Therefore, whether the boost of lactic acid bacteria contributes to the immunological response in the elderly metabolic syndrome is interesting to be studied in the future.

\subsection{YTD modulated gut microbiota and reduced plasma lipoprotein (a)}

YTD also decreased the concentration of lipoprotein (a), a well-established risk factor of cardiovascular disease and atherosclerosis. Decrease of lipoprotein (a) reflected the clinical effect of alleviating metabolic syndrome in elderly persons by YTD. Interestingly, a negative correlation was identified between lipoprotein (a) and an Acinetobacter species, suggesting a possible linkage between gut microbes and biochemical parameters. The metabolic product of Acinetobacter, an endophytic bacteria of Lycium barbarum (a major component of YTD), has antimicrobial activity against Staphylococcus aureus (29). These results further support the benefit of YTD to alleviate metabolic syndrome.

\subsection{Which component of YTD causes changes in gut microbiota}

Recent studies have found that a considerable number of Chinese herbs can directly or indirectly affect gut microbes. Coincidentally, Polygonatum sibiricum, Rehmannia glutinosa Libosch, Lycium barbarum and Radix puerariae in YTD have been proved to significantly change the intestinal microbial structure of patients with metabolic diseases in multiple studies.

Polygonatum sibiricum contains a variety of beneficial elements, such as polysaccharides, steroidal saponins, anthraquinones, alkaloids, cardiac glycosides, lignin, vitamins and amino acids. Wang YF (30) used the polysaccharide of Polygonatum sibiricum to interfere with the intestinal flora of rats with lipid metabolism disorder. Yun Nan rhizoma polygonati polysaccharide can reverse Firmicutes and Bacteroidetes, reduce the relative abundance of Proteobacteria, Lactobacillus and Psychrobacter, and show favorable effects on the composition of gut microbiota.

One of the main components of Rehmannia glutinosa Libosch is stachyose. Stachyose addition affects gut microbiota of STZ induced diabetic mice (31). Compared with the normal group, in the stachyose group, Proteobacteria $(p<0.01)$, but decreased significantly in Firmicutes $(p<0.01)$ and decreased slightly in Bacteroidetes; the numbers of bacteria significantly decreased in Lactobacillus and Bacteroides $(p<0.01)$ but significantly increased in Helicobacterium and Mycoplasma $(p<0.05)$.

The main ingredients of Lycium barbarum are lyceum barbarum polysaccharide, betaine, riboflavin, sterol, rutin and lycium, and various amino acids and trace elements (32). Liu YT (33) found that low concentration extracts of Fructus Lycii showed promoting effects on Bifidobacterium and Lactobacillus, which was better than that of the high concentration group. However, the promoting effects on enterobacteria and enterococcus were not obvious.

The main active components of Radix Puerariae are pueraria isoflavones, daidzein and puerarin 7-xyloside. $\mathrm{Xu} \mathrm{J}$ (34) examined the effect and mechanism of Gegen Qinlian decoction (GQD, a traditional Chinese herbal formula containing pueraria as the main component) on the treatment of type 2 diabetes by investigating intestinal flora composition. They found that patients who were given a high dose of GQD could significantly recover their blood sugar levels, as well as the levels of HbA1c. Analysis of gut microbiota composition found that GQD treatment changed the abundance of 47 phylotypes, 17 of which are negatively correlated with blood glucose levels, and 9 that are negatively correlated with HbA1c levels. The study confirmed that GQD could change the intestinal flora by regulating the number of probiotics in the intestinal tract, thus achieving the function of treating type 2 diabetes.

Therefore, we hypothesized that YTD may regulate the gut microbiota through one or more active ingredients of these Chinese herbs or ingredients, thus contributing to the clinical efficacy of alleviating metabolic syndrome. 
In conclusion, by analyzing the gut microbiota in elderly persons, a high inter-individual variation in gut microbiota was observed. The Chinese elderly populations employed in this study had a gut microbiota dominated by Firmicutes and Actinobacteria. Our result further highlights the distinct fecal microbiota in elderly patients with metabolic syndrome compared with healthy controls. Metabolic syndrome patients had higher relative abundance of Lactobacillus and Bifidobacterium and lower Anaerostipes and Coprococcus than healthy controls. The Chinese herbal formula Yangyin Tiluo Decoction could reduce the abundance of potentially pathogenic bacteria and lipoprotein (a), which provides an approach to treat elderly patients with metabolic syndrome. Collectively, these findings gain insights into the gut microbiota of elderly patients with metabolic syndrome, and the role of YTD in modulating gut microbiota during metabolic syndrome treatment.

\section{Acknowledgements}

The work was partly supported by Research Fund for Chinese Medicine from Administration of Traditional Chinese Medicine of Jiangsu Province (2100601).

\section{Availability of data and materials}

All data generated and analyzed during this study are included in this published article. The raw sequence reads were deposited into NCBI Sequence Read Archive (SRA) database (accession SRP118482).

\section{References}

1. Eckel RH, Grundy SM, Zimmet PZ. The metabolic syndrome. Lancet. 2005; 365:1415-1428.

2. Gami AS, Witt BJ, Howard DE, Erwin PJ, Gami LA, Somers VK, Montori VM. Metabolic syndrome and risk of incident cardiovascular events and death: A systematic review and meta-analysis of longitudinal studies. J Am Coll Cardiol. 2007; 49:403-414.

3. Musso G, Gambino R, Cassader M. Interactions between gut microbiota and host metabolism predisposing to obesity and diabetes. Annu Rev Med. 2011; 62:361-380.

4. Ley RE, Turnbaugh PJ, Klein S, Gordon JI. Microbial ecology: Human gut microbes associated with obesity. Nature. 2006; 444:1022-1023.

5. Qin JJ, Li YR, Cai ZM, et al. A metagenome-wide association study of gut microbiota in type 2 diabetes. Nature. 2012; 490:55-60.

6. Xu J, Lian FM, Zhao LH, Zhao YF, Chen XY, Zhang X, Guo Y, Zhang C, Zhou Q, Xue Z, Pang X, Zhao L, Tong X. Structural modulation of gut microbiota during alleviation of type 2 diabetes with a Chinese herbal formula. ISME J. 2015; 9:552-562.

7. Chen KC, Lu R, Iqbal U, Hsu KC, Chen BL, Nguyen PA, Yang HC, Huang CW, Li YC, Jian WS, Tsai SH. Interactions between traditional Chinese medicine and western drugs in Taiwan: A population-based study. Comput Methods Programs Biomed. 2015; 122:462-470.
8. Zhang J. The clinical study of the Nourishing-Yin-andDredging-Collateral Formula's intervention in chronic renal damage towards elderly patients with matabolic syndrome. Nanjing: Nanjing University of Chinese Medicine; 2011. (in Chinese)

9. Zhu CL, Guo HM. Research on effect of NourishingYin-and-Dredging-Collateral Formula on inflammatory mechanisms of human venous endothelial cells. Changchun Zhong Yi Yao Da Xue Xue Bao. 2014; 30:405-407. (in Chinese)

10. Expert Panel on Metabolic Syndrome of Chinese Diabetes Society. Recommendations on metabolic syndrome of Chinese diabetes society. Zhongguo Tang Niao Bing Za Zhi. 2004;12:156-161. (in Chinese)

11. Zoetendal EG, Akkermans ADL, De Vos WM. Temperature gradient gel electrophoresis analysis of $16 \mathrm{~S}$ rRNA from human fecal samples reveals stable and hostspecific communities of active bacteria. Appl Environ Microbial. 1998; 64:3854-3859.

12. Mu CL, Yang YX, Su Y, Zoetendal EG, Zhu WY. Differences in microbiota membership along the gastrointestinal tract of piglets and their differential alterations following an early-life antibiotic intervention. Front Microbiol. 2017; 8:797.

13. Caporaso JG, Kuczynski J, Stombaugh J, et al. QIIME allows analysis of high-throughput community sequencing data. Nat Methods. 2010; 7:335-336.

14. Cole JR, Wang Q, Cardenas E, Fish J, Chai B, Farris RJ, Kulam-Syed-Mohideen AS, McGarrell DM, Marsh T, Garrity GM, Tiedje JM. The Ribosomal Database Project: Improved alignments and new tools for rRNA analysis. Nucleic Acids Res. 2009; 37:D141-D145.

15. Lozupone C, Lladser ME, Knights D, Stombaugh J, Knight R. UniFrac: An effective distance metric for microbial community comparison. ISME J. 2011; 5:169172.

16. Segata N, Izard J, Waldron L, Gevers D, Miropolsky L, Garrett WS, Huttenhower C. Metagenomic biomarker discovery and explanation. Genome Biol. 2011; 12:R60.

17. Xia J, Wishart DS. Using MetaboAnalyst 3.0 for comprehensive metabolomics data analysis. Curr Protoc Bioinformatics. 2016; 55:14.10.1-14.10.19.

18. Benjamini Y, Hochberg Y. Controlling the false discovery rate: A practical and powerful approach to multiple testing. J Roy Stat Soc B Met. 1995; 57:289-300.

19. Claesson MJ, Cusack S, O'Sullivan O, et al. Composition, variability, and temporal stability of the intestinal microbiota of the elderly. Proc Natl Acad Sci USA. 2011; 108:4586-4591.

20. Milani C, Ticinesi A, Gerritsen J, et al. Gut microbiota composition and Clostridium difficile infection in hospitalized elderly individuals: A metagenomic study. Sci Rep. 2016; 6:25945

21. O'Flaherty S, Saulnier DM, Pot B, Versalovic J. How can probiotics and prebiotics impact mucosal immunity? Gut Microbes. 2010; 1:293-300.

22. Le KA, Li Y, Xu XJ, Yang WT, Liu TT, Zhao X, Tang YG, Cai D, Go VL, Pandol S, Hui H. Alterations in fecal Lactobacillus and Bifidobacterium species in type 2 diabetic patients in Southern China population. Front Physiol. 2013; 3:496.

23. Karlsson FH, Tremaroli V, Nookaew I, Bergstrom G, Behre CJ, Fagerberg B, Nielsen J, Bäckhed F. Gut metagenome in European women with normal, impaired and diabetic glucose control. Nature. 2013; 498:99-103. 
24. Larsen N, Vogensen FK, van den Berg FWJ, Nielsen DS, Andreasen AS, Pedersen BK, Al-Soud WA, Sørensen SJ, Hansen LH, Jakobsen M. Gut microbiota in human adults with type 2 diabetes differs from non-diabetic adults. Plos One. 2010; 5:e9085

25. Wang W, Chen LP, Zhou R, Wang XB, Song L, Huang S, Wang G, Xia B. Increased proportions of Bifidobacterium and the Lactobacillus group and loss of butyrateproducing bacteria in inflammatory bowel disease. J Clin Microbiol. 2014; 52:398-406.

26. Zeng H, Liu J, Jackson MI, Zhao FQ, Yan L, Combs GF Jr. Fatty liver accompanies an increase in Lactobacillus species in the hind gut of C57BL/6 mice fed a high-fat diet. J Nutr. 2013; 143:627-631.

27. Thevaranjan N, Puchta A, Schulz C, et al. Age-associated microbial dysbiosis promotes intestinal permeability, systemic inflammation, and macrophage dysfunction. Cell Host Microbe. 2017; 21:455-466.

28. Tsilingiri K, Barbosa T, Penna G, Caprioli F, Sonzogni A, Viale G, Rescigno M. Probiotic and postbiotic activity in health and disease: Comparison on a novel polarised exvivo organ culture model. Gut. 2012; 61:1007-1015.

29. Du XN, Xu HJ, Huang PP, Dai JX. Diversity and antimicrobial activity of endophytic bacteria isolated from
Lycium barbarum of Ningxia. Wei Sheng Wu Xue Tong Bao. 2015; 42:1779-1787. (in Chinese)

30. Wang YF. Effects and mechanisms of polysaccharide from Polygonatum sibiricum on lipid metabolism disorder in rats. Yunnan Traditional Chinese Medicine College, 2017. Thesis. (in Chinese)

31. Wang L. Effects of stachyose in rehmannia on intestinal microflora in diabetic mice. Peking Union Medical College, 2013. Thesis. (in Chinese)

32. Lv BY, Fan RX.The composition and function of Lycium barbarum L. Hebei Zhong Yi Yao Xue Bao. 1998; 13:2930. (in Chinese)

33. Liu YT, Xu W, Xin Y, Li XL. Regulating effects of Fructus Lycii extracts on dysbiosis of intestinal flora of mice. Tian Ran Chan Wu Yan Jiu Yu Kai Fa. 2015; 27:12701272,1295. (in Chinese)

34. Xu J, Lian F, Zhao L, Zhao Y, Chen X, Zhang X, Guo Y, Zhang C, Zhou Q, Xue Z, Pang X, Zhao L, Tong X. Structural modulation of gut microbiota during alleviation of type 2 diabetes with a Chinese herbal formula. ISME J. 2015; 9:552-562.

(Received March 12, 2018; Revised June 13, 2018; Accepted June 24, 2018) 